\title{
MULTIPLE CHOICE QUESTION BASED ON NEWER ANTI-EPILEPTIC DRUGS
}

Q.1 A 6 year old boy suffering from Gilbert syndrome, presented with seizure characterized by sudden behavioural arrest, unresponsiveness, and a blank stare typically lasting for a few seconds to half a minute and 1 episode of generalized seizure. With The ictal EEG findings consist of $3 \mathrm{~Hz}$ spike and wave complexes with occasional occipital paroxysmal bursts preceding the generalized discharges

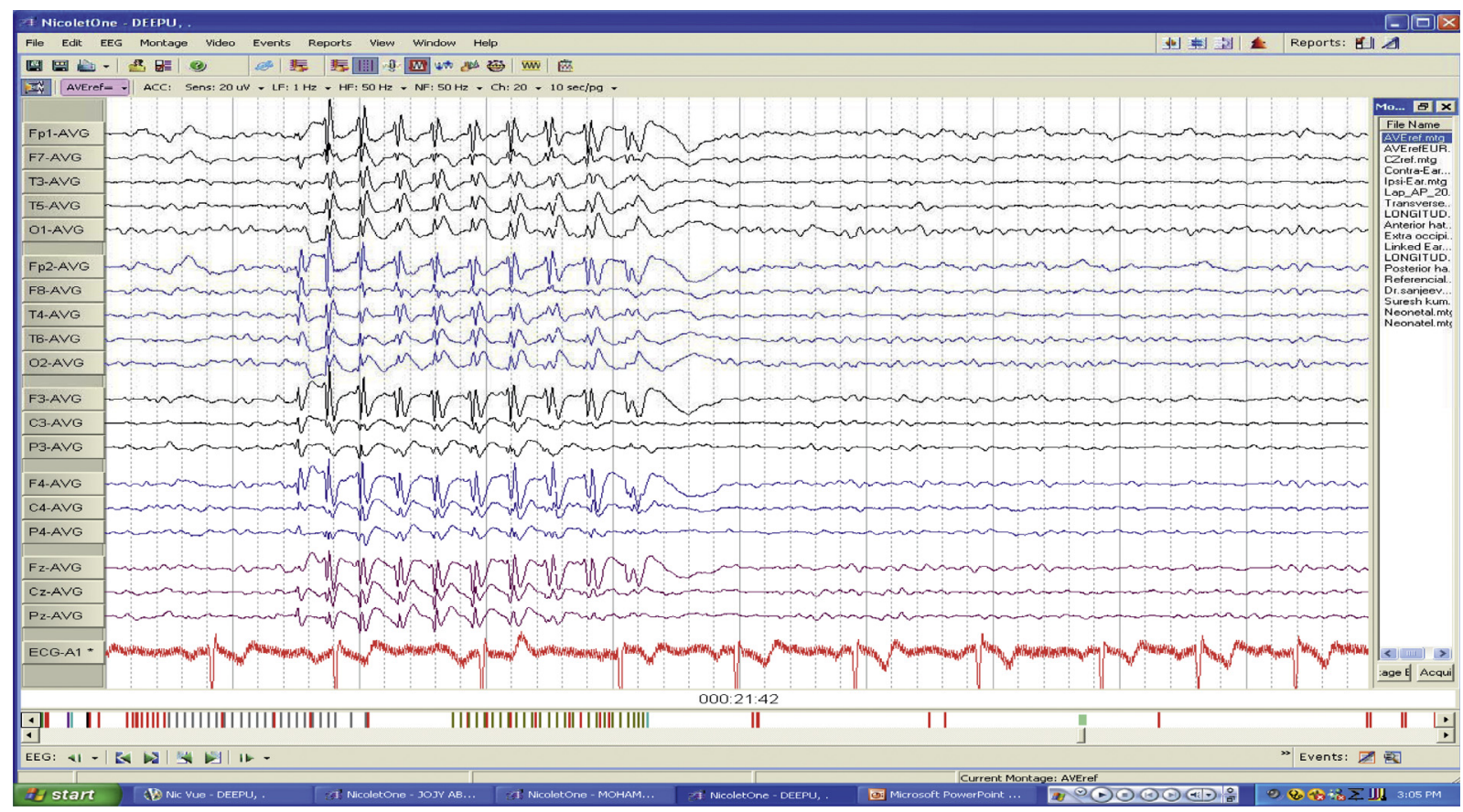

Which of the following drug is most useful in this patient for seizure control?
A) Valproic acid
B) Levitiracetam
C) Ethosuximide
D) Oxycarbazepine

Q.2 A 70-year-old woman with a history of osteoporosis presents with newly diagnosed partial epilepsy believed to have resulted from an old right middle cerebral artery infarct. She was started on phenytoin in the emergency department 1 month ago. She is otherwise healthy and takes no medications other than aspirin, simvastatin, and a bisphosphonate.

Which of the following anticonvulsants is most appropriate for long-term seizure control in this patient?
A) Carbamazepine
B) Lamotrigine
C) Phenytoin
D) Valproic Acid

Q.3 30 year old business man presented with new-onset complex partial seizure with focal left temporal sharp waves, with atopy, allergy, and migraine, started on carbamazepine developed rashes on day 3 . Which of the following newer antiepileptic is useful for this patient?
A) Levitiracetam
B) Lacosamide
C) Lamotrigine
D) Topiramate 
Q.4 26 year old female, teacher by occupation, having seizure since the age of 21 , she was under valproate. Once she got married and planning for baby she was switched over to lamotrigine. She delivered a healthy baby, now worried about whether she can feed the baby? What action would be best in this patient?
A) Change to Topiramate and continue breast feed
B) Get back to sodium valproate and continue breast feed
C) Stop Breastfeed and advise Top feed for the baby
D) Continue on lamotrigine and continue to breast feed

Q.5 8 year old boy with psychomotor retardation and frequent drop attacks started at the age of 5 years, with multiple seizure types including GTCS, myoclonic jerks, typical absence seizures, with has a Characteristic EEG abnormalities suggestive of Lennox-Gastaut syndrome LGS. He had poor school performance and learning disability, MRI showed bilateral perisylvian polymicrogyria. Her routine workup showed prolonged $\mathrm{Q}-\mathrm{R}$ interval and P-R interval in ECG.
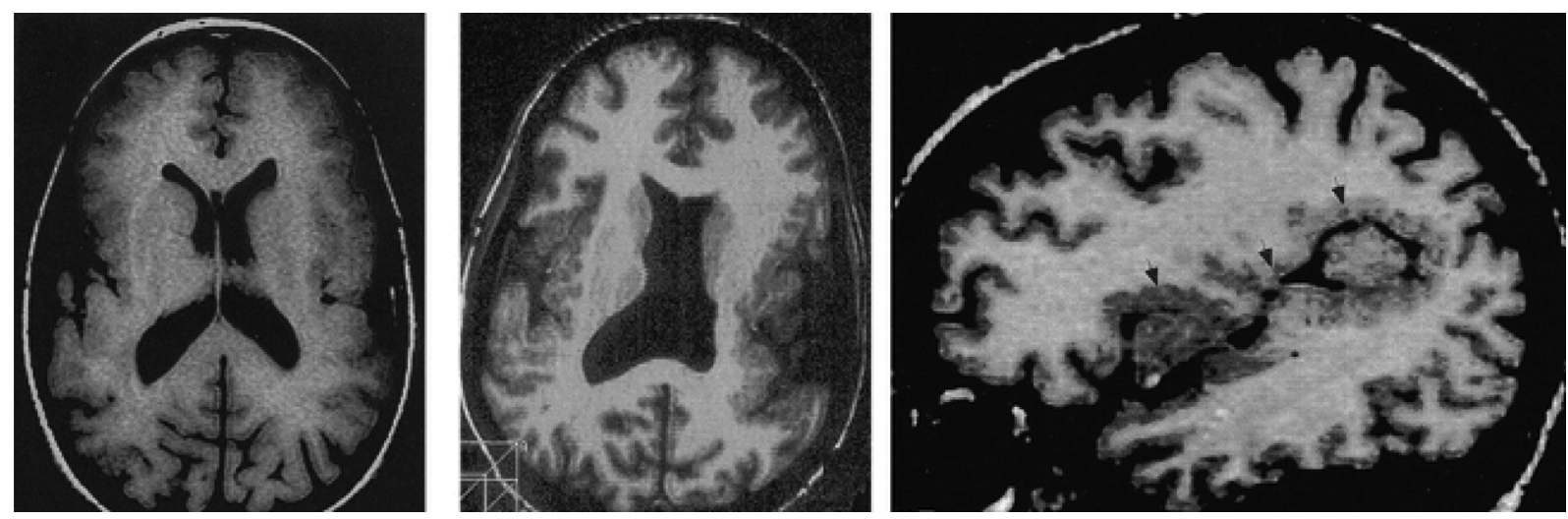

http://www.ajnr.org/content/20/10/1814.full

Which AED is effective and optimal in this patient?
A) Lacosamide
B) Topiramate
C) Rufinamide
D) Ezogabine

Q.6 30 year old bank employer, had head trauma SDH operated and had seizure, RT focal with secondary generalization, even after discharge had two to three episodes of seizure in a week. She was under Oral contraceptive pills. Which of the following will be safe and effective?
A) Carbamazepine
B) Lamotrigine
C) Levitiracetam
D) Oxcarbazepine

Q.7 A 17 year old girl had acute tubular necrosis and renal failure; elevated renal parameters developed spontaneous intracranial haemorrhage and had RT focal seizure with secondary generalization, which of the following drug is safe in this patient.
A) Levitiracetam
B) Topiramate
C) Lacosamide
D) Tiagabine 
Q.8 Which of the following newer AED is approved by FDA for adjunctive use partial epilepsy which has shown to prevent kindling phenomenon and reduce neuronal excitability?
A) Peramapanel
B) Rufinamide
C) Lacosamide
D) Ezogabine

Q.9 A 32 year old gentleman, working as public relation officer in a MNC, was under treatment for complexpartial seizure with comorbid vertigo \& dizziness, for 4 year duration. Due to poor control of seizures, carbamazepine was stepped up to max dose of $1200 \mathrm{mg}$. What newer AED will be safe adjunctive to add without producing much adverse effects to control his seizure?
A) Lacosamide
B) Lamotrigine
C) Levitiracetam
D) Zonisamide

Q.10 83 year old gentlemen presented with new-onset seizure, with co-morbid, diabetic, hypertension, coronary artery disease, with recent decline in cognition. Which antiepileptic will be safe first line monotherapy for this patient?
A) Topiramate
B) Tiagabine
C) Oxcarbazepine
D) Levitiracetam

Dr. Lakshmi Narasimhan DM (Neurology), DNB (Neurology)

Professor of Neurology

Institute of Neurology

Madras Medical College

Chennai - 600003.

Mobile : +91-94443-80409

Landline : +91-44-2461-8442

\section{Answers}

\section{Epilepsy Quiz Answers:}

\section{Answer B. Levitiracetam}

Explanation/comment: The above described symptoms and EEG is characteristic of absence seizure and the following drugs are effective in treating Absence seizure Valproic acid, Ethosuximide, benzodiazepines, Levitiracetam, and Phenobarbital.

Though Valproic acid is effective in absence seizure it activates CYP: 2C9, epoxide hydrolase and uridine diphosphate glucuronosyltransferase in hepatic metabolism, hence not ideal in this child with gilbert syndrome.

Ethosuximide although the drug of choice for absence seizure it acts by blocking the T-type calcium channels in thalamic neurons in a magnitude effective enough to reduce the amplitude of threshold spikes preventing bursting and absence seizure activity. Ethosuximide does not block fast voltage gated sodium channels involved in generalized seizure, so ethosuximide is ineffective in case of co-existing generalized tonic-clonic seizures.

There are class IV reports demonstrating that carbamazepine, oxcarbazepine, phenobarbital, phenytoin, tiagabine, and vigabatrin may worsen or precipitate absence seizures.

Reference: Stuart M. Cain, Terrance P. Snutch T-type calcium channels in burst-firing, network synchrony, and epilepsy, Volume 1828, Issue 7, July 2013, Pages 1572-1578; Biochimica et Biophysica Acta (BBA) - Biomembranes. 
Explanation/comment: The issues in this patient is

1. Decreased bone marrow density.

2. Post stroke seizure.

3. Interaction of drugs taken for above comorbid conditions.

Antiepileptic drugs that are inducers of the hepatic cytochrome P450 enzyme system (including phenytoin, phenobarbital, primidone, and carbamazepine) increase the metabolism of vitamin D. Valproic acid is an inhibitor of the cytochrome P450 system but is also associated with altered bone metabolism and decreased bone mass. Lamotrigine has not been associated with bone loss and may be a better choice in this patient.

Reference: Nature Reviews Neurology 10, 485 (2014) doi: 10.1038/nrneurol.2014.154 Published online 19 August 2014.

\section{Answer D. Topiramate}

Explanation/comment: When we go by studies, "The Standard and New Antiepileptic Drugs (SANAD) study" showed Lamotrigine had the longest time to treatment failure interval, and carbamazepine had the shortest time interval to failure. But looking into the co-morbid status is also vital when choosing AED.

Newer AED potential to cause rash includes, Rufinamide, zonisamide (sulpha allergy), oxcarbazepine, felbamate, and ethosuximide, lamotrigine. Topiramate is ideal for patient with co-morbid migraine. Patient with chronic pain disorder gabapentin and pregabalin is preferred.

While choosing AED, avoid drugs which can worsen the co-morbid condition, like avoid topiramate and zonisamide for patients with nephrolithiasis. Patient with atopic disorder are prone for developing rash with Lamotrigine and carbamazepine. Topiramate and zonisamide can increase weight that is already obese. Levitiracetam, which aggravated irritability and anger outburst.

Reference: CONTINUUM: Lifelong Learning in Neurology: June 2010 - Volume 16 - Issue 3, Epilepsy pp 121-135 doi: 10.1212/01.CON.0000368235.64857.cf

\section{Answer D. Continue on lamotrigine and continue breast feed}

Explanation/comment: While deciding the potential risk vs. benefit of breast feeding in nursing infant, conventional drugs like phenytoin, carbamazepine and sodium valproate are considered safe because they have less penetrance into breast milk.

Gabapentin, lamotrigine, and topiramate, levitricetam, penetrate in significant amount there are insufficient data to determine whether or not these drugs can clinically affect infants. However mothers taking, Phenobarbitone, benzodiazepine, are advised to follow a mixed regimen of breastfeeding and Top feeding for the first 5 to 7 days postpartum, because of the delayed elimination of AED.

Reference: Harden CL, Hopp J, Ting TY et al. Practice parameter update: management issues for women with epilepsy - focus on pregnancy (an evidence-based review): III. Vitamin K, folic acid, blood levels, and breastfeeding: report of the Quality Standards Subcommittee and Therapeutics and Technology Assessment Subcommittee of the American Academy of Neurology and American Epilepsy Society. Neurology 2009; 73: 142-149.

Johannessen SI, Helde G, Brodtkorb E. Levitiracetam concentrations in serum and in breast milk at birth and during lactation. Epilepsia 2005; 46: 775-777.

\section{Answer B. Topiramate}

Explanation/comment: Among newer antiepileptic drugs, Lamotrigine, topiramate, rufinamide, clobazam, and felbamate are effective in treating Lennox-Gastaut Syndrome. But rufinamide and Ezogabine are potential to cause Q-T prolongation syndrome and Lacosomide causes $\mathrm{P}-\mathrm{R}$ interval prolongation. Base line EKG is must before treating LGS, with rufinamide and Ezogabine. During the course of treatment when the QTc falls below $300 \mathrm{msec}$, the risk of ventricular fibrillation is more. Don't choose Rufinamide for patients with familial history of short QTc interval. 
Prolongation of the P-R interval on the EKG was noted to occur in a dose-dependent manner with lacosamide. A baseline and post-treatment EKG is suggested especially in patients with cardiac arrhythmias, cardiomyopathies, and drugs with cardiac conduction effects.

Reference: Practical Neurology Vol. 8, No. 8, November/December 2009.

6. Answer C. Levitiracetam

Explanation/comment: AEDs that can adversely affect hormonal contraceptives via Hepatic Cytochrome P450 enzyme induction are termed as Enzyme Inducing Anti-Epileptic Drug (EIAED). Plasma concentration of, oxcarbazepine, Felbamate, Topiramate ( $>200 \mathrm{mg} / \mathrm{dl})$, Lamotrigine are reduced by $40 \%-60 \%$ in women taking oral contraceptive pills. Drugs like Gabapentin, Lacosomide, Levitiracetam, pregabalin, Tiagabine, zonisamide found to have no interaction with hormonal contraceptives.

In a patient who is on EIAED there is a risk of low hormone levels during the pill-free period which can lead to unplanned pregnancy. Hence, an alternative could be to use regimes with short hormonefree intervals (4-5 days) or continuous dose medication for three months or so (tricycling).

Termed as "TRICYCLE CONTRACEPTION". The Lamotrigine dose may need to be reduced during the week of withdrawal bleeding.

Reference: Ann Indian Acad Neurol 2015;18:278-283.

7. Answer D. Tiagabine

Explanation/comment: Of the newer AEDs, only tiagabine undergoes extensive liver metabolism making it possible to use in regular doses in renal failure. On the contrary, gabapentin and vigabatrin are excreted almost exclusively by the kidneys with negligible liver metabolism. Topiramate, when not used concomitantly with enzyme-inducing drugs, is almost $90 \%$ eliminated directly through the kidneys.

Important dose adjustments will be necessary when using gabapentin, vigabatrin, or topiramate in patients with moderate to severe renal insufficiency. Other newer AEDs are eliminated by combination of hepatic metabolism and /or direct renal excretion.

Figure 1 summarize the relative contribution of renal excretion and hepatic metabolism in the elimination of the AEDs.

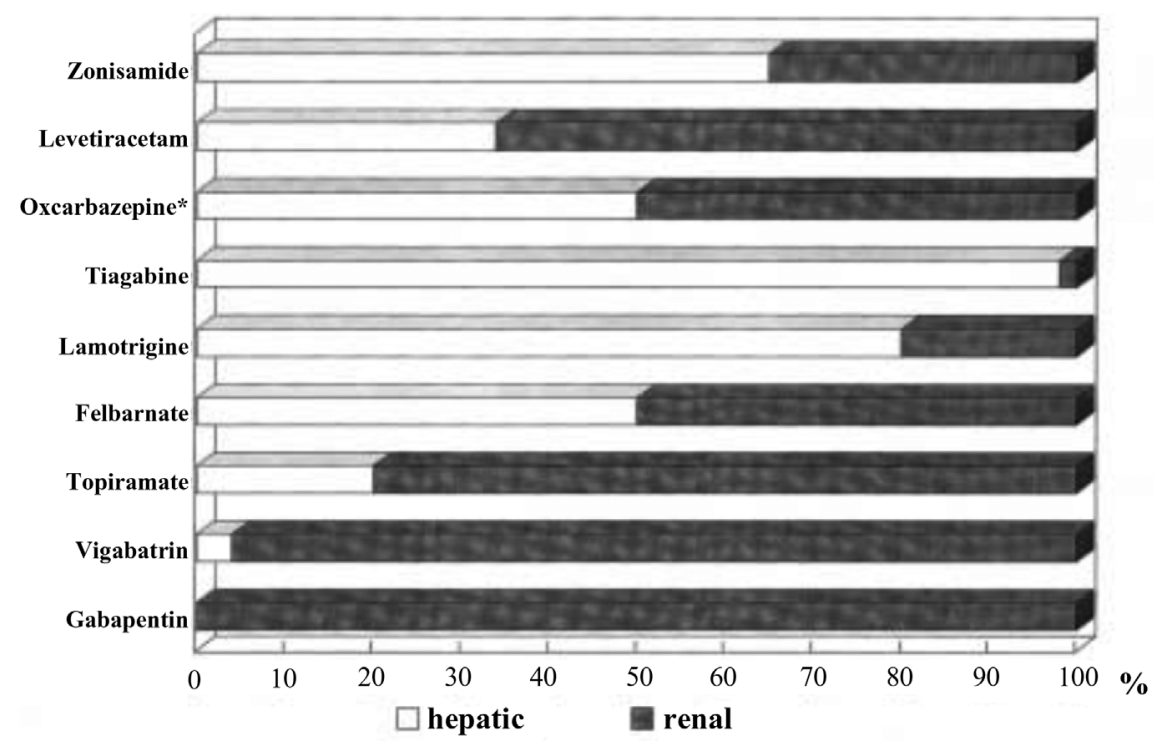

Reference: Jorge J. Asconapé, M.D. Seminars in Neurology/Volume 22, Number 12002. 
8. Answer A. Perampanel

Explanation/comment: MCRDBPC clinical trials done, demonstrated the beneficial and efficacy of newer AED in adult onset partial epilepsy syndrome. Out of which four newer AED, with unique mechanism of action is found useful in treating partial-onset seizure.

The following table gives a brief note on the mechanism of action of these newer AED.

\begin{tabular}{|l|l|}
\hline Perampanel & $\begin{array}{l}\text { Acts on the AMPA receptors involved in generation of kindling phenomenon. } \\
\text { It inhibits the increases in free calcium ion concentrations in cortical } \\
\text { neurons, reducing neuronal excitability. }\end{array}$ \\
\hline Rufinamide & $\begin{array}{l}\text { Acts by maintaining the sodium channel in a prolonged inactive state to } \\
\text { inhibit the repetitive firing of the sodium dependent action potentials. }\end{array}$ \\
\hline Lacosamide & $\begin{array}{l}\text { Acts selectively enhance the slow inactivation of voltage gated sodium } \\
\text { channel. }\end{array}$ \\
\hline Ezogabine & $\begin{array}{l}\text { Acts as a neuronal potassium channel opener to stabilize neuronal membrane } \\
\text { during repetitive firing. }\end{array}$ \\
\hline
\end{tabular}

Reference: Jacqueline A. French, MD, FAAN; Deana M. Gazzola, MD; Continuum (Minneap Minn) 2013;19(3):643-655.

\section{Answer C. Levitiracetam}

Explanation/comment: While choosing AED in combinations the drug-drug interaction and their side effect profile tend to increase. The following combinations, which are commonly employed, are notorious for exacerbating these symptoms of dizziness, imbalance, and diplopia.

(1) Carbamazepine and Lamotrigine

(2) Carbamazepine and lacosamide

(3) Oxcarbazepine and lacosamide

(4) Lamotrigine and lacosamide.

\begin{tabular}{|l|c|c|}
\hline \multicolumn{1}{|c|}{ Newer AED } & $\begin{array}{c}\text { Effect of newer AED } \\
\text { on carbamazepine }\end{array}$ & $\begin{array}{c}\text { Effect of } \\
\text { carbamazepine on } \\
\text { Newer AED }\end{array}$ \\
\hline Lacosamide & None & Decrease \\
\hline Lamotrigine & None & Decrease \\
\hline Levitiracetam & None & None \\
\hline Zonisamide & None & Decrease \\
\hline Topiramate & None & Decrease \\
\hline Rufinamide & Decrease & Decrease \\
\hline Vigabatrin & None & None \\
\hline
\end{tabular}

Reference: Jacqueline A. French, MD, FAAN; Deana M. Gazzola, MD; Continuum (Minneap Minn) 2013;19(3):643-655.

\section{Answer D. Levitiracetam}

Explanation/comment: In treating elderly with epilepsy, understanding their pharmacokinetics and physiology of the other system should be taken care. Older drugs with high protein bound tend to have higher level of active fraction producing adverse side effects. Effects on cognition, alertness, and gait stability are of particular concern.

Among newer AED. Oxcarbazepine has been associated with hyponatremia in older individuals, particularly in those patients on diuretics. Levitiracetam is a broad-spectrum without much adverse effects, though it produces dizziness and somnolence. 
Topiramate has gained FDA approval as first line monotherapy in epilepsy, but it has an unfavourable cognitive profile in comparison with Lamotrigine. Both Lamotrigine and topiramate can be used in elderly but need slow titration of dose, hence not useful where rapid control of seizure is needed.

Tiagabine, zonisamide, pregabalin are recommended as adjunctive AED and not as first line.

The most common adverse effect with tiagabine is dizziness.

Reference: Gina Mapes Jetter, M.D., 1 and Jose E. Cavazos, M.D., Ph.D., Seminars In Neurology/ Volume 28, Number 32008. 